\title{
Agroforestería como alternativa de desarrollo sostenible en el territorio indígena de Salitre, zona de amortiguamiento del Parque Internacional la Amistad ${ }^{1}$
}

\author{
K. Melissa Castillo-Rojas* \\ (iD https://orcid.org/0000-0002-1552-3627
}

Recibido: 19 de noviembre, 2019 - Aceptado: 20 de febrero, 2020

\begin{abstract}
RESUMEN
En este artículo se plantea que la agroforestería cierra la brecha entre la conservación y el desarrollo sostenible en la comunidad rural de Salitre y, por lo tanto, podría renombrarse como agroforestería social. En este territorio indígena hay una falta de empleo que, en consecuencia, conduce a la pobreza extrema; además de esto, hay prácticas inapropiadas en el uso de la tierra, tales como el uso del fuego para el control de malezas, así como el conflicto entre las poblaciones indígenas y no indígenas. Se observa la necesidad de establecer sistemas agroforestales en el área,
\end{abstract}

\section{Formato de citación según APA}

Castillo-Rojas, M. (2020). Agroforestería como alternativa de desarrollo sostenible en el territorio indígena de Salitre, zona de amortiguamiento del Parque Internacional la Amistad. Revista Espiga, 19 (39), 63-76.

\section{Formato de citación según Chicago-Deusto}

Castillo-Rojas, Melissa (2020). «Agroforestería como alternativa de desarrollo sostenible en el territorio indígena de Salitre, zona de amortiguamiento del Parque Internacional la Amistad». Revista Espiga 19, n. ${ }^{\circ} 39$ (enero-junio, 2020): 63-76.

* Estudiante de Manejo de Recursos Naturales en la Universidad Estatal a Distancia (UNED), en el Centro Universitario de Buenos Aires, provincia de Puntarenas, Costa Rica. El presente artículo fue escrito posteriormente a la realización de su Trabajo Final de Graduación (TFG) para optar por el grado de bachillerato. Es el resultado de la investigación realizada propiamente en el territorio indígena de Salitre, cuyo fuerte contenido social fue valorado por el Comité Editorial de la Revista Espiga para su publicación. Correo: melissak962010@gmail.com

1. La autora extiende un agradecimiento extraordinario a los pobladores de Salitre por su valiosa participación en la investigación llevada a cabo, en especial a Guillermo Elizondo Ortiz, administrador del Centro Cultural Bribripa. También, agradece al Centro Nacional de Alta Tecnología (CeNAT-CONARE) por la colaboración y financiamiento en el desarrollo de la investigación, en especial al ingeniero Allan Campos Gallo, director del Área de Gestión Ambiental, así como a la magíster Ana Victoria Wo Ching por sus apreciables aportes y apoyo incondicional. 
ya que este tipo de modelo de producción es una mejor alternativa para el desarrollo rural en un sentido amplio. El artículo también destaca los sistemas tradicionales de producción indígena, ya que se argumenta que los bribris del sureste de Costa Rica fundaron una región basada en el uso y la conservación de los recursos forestales y la diversidad cultural. La agroforestería social es clave para comprender los sistemas de gestión de recursos naturales, las prácticas y los usos de la tierra, desde un punto de vista cultural. Por último, varias especies de plantas que crecen en Salitre pueden utilizarse como índice de conservación e interacción cultural, como alternativa de producción y como medio para lograr la seguridad alimentaria.

Palabras clave: Sistemas agroforestales, área protegida, integración cultural, seguridad alimentaria, conservación.

\section{Introducción}

En Costa Rica, habitan ocho pueblos indígenas que representan el 2\% de la población ${ }^{2}$. Al respecto, el cantón de Buenos Aires, en Puntarenas, es multiétnico, pues hay cuatro grupos indígenas: bruncas, térrabas, bribris y cabécares. Dentro de sus límites existen seis territorios indígenas, entre estos Salitre ${ }^{3}$.

El territorio de Salitre fue establecido en 1982 y cuenta con alrededor de 1285 personas indígenas ${ }^{4}$. Es uno de los 23 territorios indígenas de Costa Rica y en él destacan los poblados de Olán, Sipar, Palmital, Río Azul, Yeri, Santa Candelaria, Salitre Centro, Puente y Alto Calderón. Su extensión es de 11700 hectáreas ${ }^{5}$. Es el más pequeño de los tres territorios bribri-cabécar de Buenos Aires y el más cercano a su cabecera principal. Los bribris llegaron aquí hace aproximadamente 150 años, provenientes de Talamanca, principalmente de las áreas de Alto Lari, Dpari y Suewebeta, todas ubicadas en el Parque Internacional La Amistad ${ }^{6}$.

Salitre posee gran importancia, dada su tenacidad en mantener su cultura ancestral y por su tradición de sistemas de policultivos. Estos son llamados skönwak, que significa «de nosotros», «lo propio» o «de nuestra gente» y con estos se ha logrado alcanzar una verdadera conexión con su medio natural ${ }^{7}$.

2. Ernesto Rivera, «Territorio indígena de Salitre», Semanario Universidad, 09 de julio 2014, acceso: 16 de marzo, 2020, https://historico.semanariouniversidad.com/page/322/?q=como-fazer-convites-gratisGRAFICA-DOS-CONVITES-como-imprimir-convites-de-casamento $\& \mathrm{n}=22514 \& \mathrm{p}=\mathrm{Cultura} \& \mathrm{jwsourc}$ $\mathrm{e}=\mathrm{cl}$

3. Federico Guevara Víquez, Cronología básica de los pueblos indigenas de Costa Rica: Desde los inicios del siglo XVI hasta el año 2000 (San José: Comisión Siwä Pakö, 2011), acceso: 16 de marzo, 2020, https:// es.scribd.com/doc/77239956/Cronologia-Basica-de-Los-Pueblos-Indigenas-CR

4. Edgar Silva de la Fuente, Vera L. Salazar Espinosa y Hugo Lázaro Estrada, Información sobre los pueblos indígenas de Costa Rica como insumo para el Proyecto Regional de Manejo Integrado de Ecosistemas por Pueblos Indigenas y Comunidades de Centroamérica (Cooperación Técnica ATN-JF-7695-BID, 2003), acceso: 16 de marzo, 2020, http://pueblosindigenas.odd.ucr.ac.cr/images/documentos/pdf/ UNPAN027540.pdf

5. Rivera, «Territorio indígena de Salitre», 2014.

6. Sistema Nacional de Áreas de Conservación, Plan de Manejo Parque Internacional La AmistadTalamanca (San José: MINAET, 2012), 24-28.

7. Carlos Borge, El policultivo indígena de Talamanca y la conservación de la naturale$z a$ (Heredia: INBio, 2011), acceso: 16 de marzo, 2020, https://espanol.free-ebooks.net/ebook/ El-Policultivo-Indigena-de-Talamanca-y-la-Conservacion-de-la-Naturaleza/pdf?dl\&preview 
Las familias bribris de Salitre manejan una producción agrícola de autoconsumo, basada en el cultivo de granos básicos (maíz, frijol), banano, cacao, pataste, plátano, yuca y frutales, combinado con la cría de gallinas, cerdos y ganadería de leche. Con dichas actividades subsisten y satisfacen sus necesidades alimenticias básicas, además de que queda algún excedente mínimo para comercialización local o para trueque, con lo cual generan ingresos. Sin embargo, las técnicas que utilizan para el desarrollo de actividades productivas son poco sostenibles, pues están basadas en sistemas de monocultivo con poca asesoría técnica; esto es resultado de las políticas asistencialistas del Estado que impulsa los monocultivos. Aunado a esto, se utiliza el fuego para el control de malezas y las áreas de cultivo se aprovechan de forma intensiva, lo que conduce al agotamiento del suelo y deja las parcelas sin capacidad para producir. Esto obliga a derribar el bosque para abrir nuevas áreas de producción, con la consecuencia de la pérdida de conectividad y aumenta la matriz agrícola en el paisaje ${ }^{8}$.

Una situación lamentable es observar cómo los potreros y los campos de cultivo de granos básicos se han convertido en terrenos infértiles. Además, la situación en Salitre es preocupante por el conflicto de la tenencia de la tierra entre indígenas y no indígenas; aunque la legislación protege el territorio, en un momento dado se permitió la venta de tierras indígenas a personas no indígenas, lo que ha hecho que algunas personas acaparen terrenos dentro de las comunidades, en grandes fincas ${ }^{9}$.

Cabe destacar que la pobreza extrema de algunos indígenas bribris de Salitre, ha provocado cambios en su actitud tradicional y aptitud cultural de uso y manejo sostenible de los recursos naturales.

Su modo de vida giraba en torno a la subsistencia agrícola tradicional, mediante la producción de cacao y banano. No obstante, la presión sobre los recursos naturales cada vez es mayor y con esto el desempleo se ha incrementado, lo cual obliga a muchos jóvenes a abandonar la zona en busca de nuevas oportunidades. El desequilibrio de la propiedad también figura como factor de incertidumbre, el deterioro en los niveles de vida es cada vez más notable, el subsistir se ha vuelto difícil y la pérdida de valores y tradiciones culturales es evidente. Cada una de estas cuestiones se resume en la falta de alternativas económicas y ambientales sostenibles.

Muchas de las personas que se encuentran en condición de desempleo, en especial jóvenes, apoyan ciertos proyectos de desarrollo que afectan la conservación del bosque y el futuro de sus propias comunidades, proyectos encaminados al divisionismo por la injerencia de foráneos dentro del territorio ${ }^{10}$.

8. R. Martínez Castillo, «La estructura cultural indígena en Talamanca», Revista Praxis, 57 (2004): 42-47; C. Campregher, «Conservación de la diversidad bio-cultural en Costa Rica: Comunidades indígenas y el ambiente», Revista Cuadernos de Antropología 21, n. ${ }^{\circ} 14$ (2011): 3-20.

9. Krissya León, «Descuido en zona indígena de Salitre sigue sumando décadas», CRHoy. com, 03 de marzo 2018, acceso: 16 de marzo, 2020, https://www.crhoy.com/nacionales/ descuido-en-zona-indigena-salitre-sigue-sumando-decadas/

10. Sistema Nacional de Áreas de Conservación, Plan de Manejo 2012, 26. 
Asimismo, los tratados de libre comercio han otorgado facilidades a las compañías madereras, por lo que existe presión para obtener tierras en las partes montañosas ${ }^{11}$. La tendencia a alquilar sus tierras a foráneos y a vivir del bajo alquiler, ha hecho que la población indígena no le adjudique la importancia que se merece al impacto ambiental generado por la aplicación de insumos agrícolas y por otras prácticas no acordes con la tradición indígena ${ }^{12}$.

Debido a la problemática que surge en el área de estudio, resulta de suma necesidad que las personas indígenas dueñas de fincas en Salitre apoyen alternativas de desarrollo sostenible, tales como modelos de producción agrícola en la modalidad de sistemas agroforestales (SAF).

En el presente artículo se ha propuesto determinar la agroforestería como un puente entre la conservación y el desarrollo sostenible de la comunidad rural de Salitre, redefiniéndola como «agroforestería social», la cual forma parte de un enfoque holístico y sistémico que busca la integración del capital natural al capital cultivado o doméstico; o sea, que integra los recursos genéticos silvestres con las opciones de desarrollo rural ${ }^{13}$.

\section{Contexto}

El territorio indígena de Salitre (figura 1) se localiza a los $9^{\circ} 11^{\prime} 28,8^{\prime \prime}$ de latitud norte y $83^{\circ} 11^{\prime} 11,4$ " de longitud oeste, pertenece al cantón de Buenos, provincia de Puntarenas, limita geográficamente con el Parque Internacional La Amistad y con el territorio indígena de Cabagra y Ujarrás ${ }^{14}$.

\section{Importancia de establecer sistemas agroforestales en Salitre}

A raíz de lo que se expone, se debe destacar que Salitre no representa únicamente una problemática social, sino también económica, ambiental y cultural. Esto se ha evidenciado a través de la observación de las necesidades de los habitantes del territorio indígena, quienes se verían sumamente beneficiados al adoptar alternativas de producción sostenible y compatibles con su cosmovisión; en otras palabras, que se relacionen estrechamente con su experiencia, su cultura y su respeto al bosque, específicamente con el establecimiento de modelos de producción agrícola con valor ecológico.

Otra de las cuestiones por tomar en consideración es que la parte alta del territorio indígena se ubica dentro de la zona de amortiguamiento del Parque Internacional La

11. Sandra Candela et al., «Formas y efectos de la gobernanza forestal en los territorios indígenas bribri y cabécar de Alta Talamanca, Costa Rica», Revista Agroforestería en las Américas, 57 (2013): 85-92.

12. Sistema Nacional de Áreas de Conservación, Plan de Manejo 2012, 26.

13. Rodrigo Arce y Alfredo García, «La agroforestería social, un nexo entre la conservación y el desarrollo sostenible», Revista Agroforestería en las Américas, 4 (s.f): 16.

14. Rivera, «Territorio indígena de Salitre», 2014. 


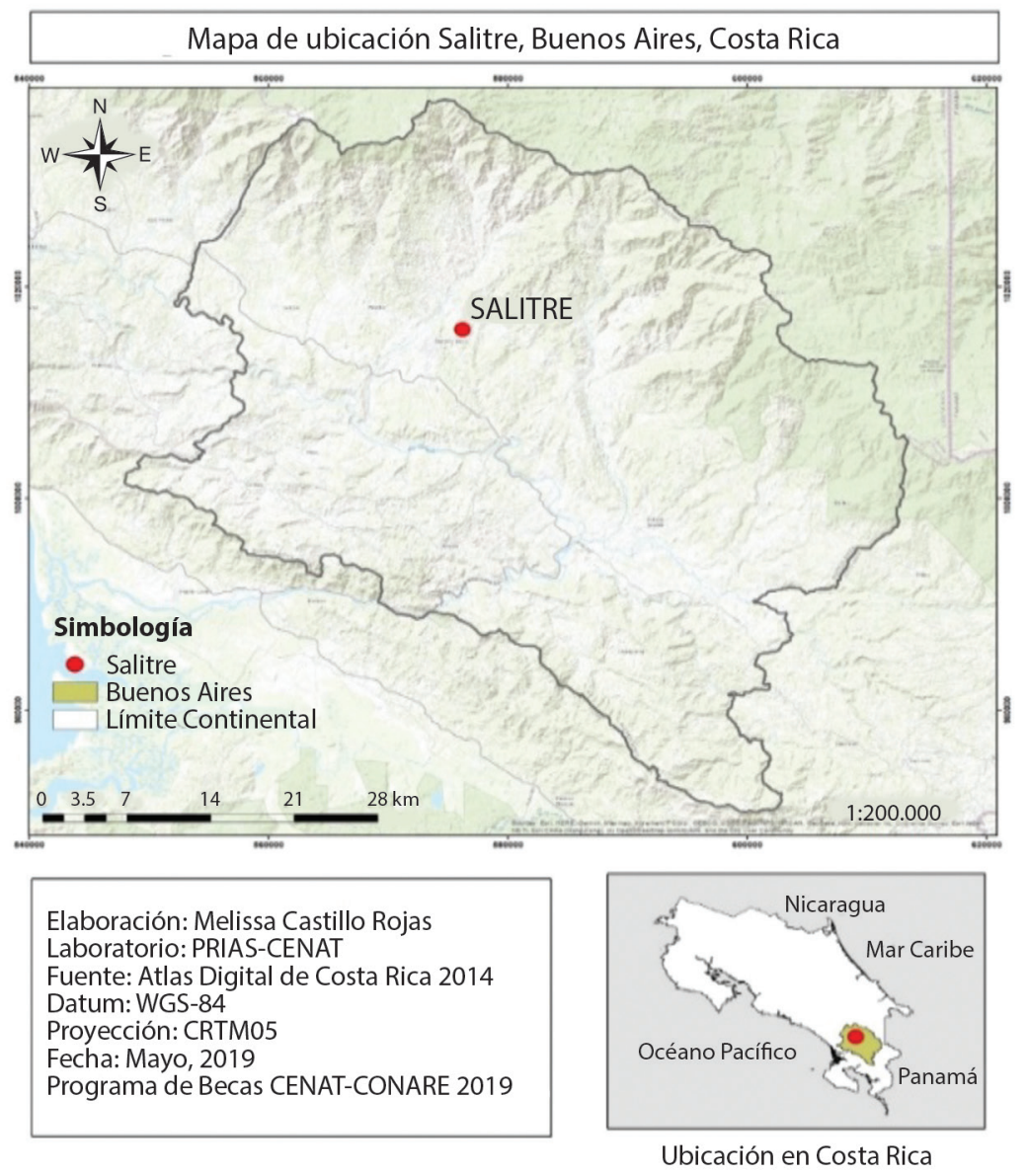

Figura 1. Ubicación de Salitre, en el cantón de Buenos Aires, Puntarenas (Costa Rica). Fuente: Elaboración propia de la autora, con base en el Atlas Digital de Costa Rica 2014.

Amistad (PILA), lo que la convierte en una excelente oportunidad y, a la vez, en un desafío para los esfuerzos de conservación en las áreas protegidas y en el paisaje productivo ${ }^{15}$.

Debido a que se cuenta con esta reserva de biosfera, como lo es el PILA, tanto área protegida como comunidad local deben poner al mismo nivel el desarrollo rural y la preservación de los recursos naturales y, por ende, el mantenimiento de los procesos ecológicos esenciales. Indudablemente, solo se podrá contar con una población activa en acciones de conservación si sus necesidades básicas han sido satisfechas ${ }^{16}$.

15. Fomento para el Medio Ambiente Mundial, «Comunidades indígenas y biodiversidad», (s.f), acceso: 16 de marzo, 2020, https://www.thegef.org/sites/default/files/publications/Indigenous-PeopleSpanish-PDF_0.pdf

16. Arce y García, Revista Agroforestería en las Américas, 4 (s.f):16. 
La agroforestería ha sido definida como un sistema de manejo dinámico y ecológico de los recursos naturales que, a través de la integración de árboles en las fincas y en los paisajes agrícolas, por ejemplo mediante la producción de productos agrícolas en los bosques, vendría a diversificar y sustentar la producción, con el fin de generar beneficios sociales, económicos y ambientales en los pobladores de Salitre ${ }^{17}$.

Ahora bien, la agroforestería puede convertirse en un método para que los agricultores logren cultivos más diversos, productivos y rentables; del mismo modo, para que también contribuyan con la protección de los recursos naturales, al evitar la erosión del suelo y reducir la dependencia de los bosques ${ }^{18}$.

En síntesis, la protección de bosques en sistemas agroforestales permitiría conectar zonas de bosque cercanas a las fincas, por medio de la vegetación que exista entre ellas. Kaimowitz ${ }^{19}$ afirma que estas áreas boscosas funcionarían como corredores biológicos, puesto que propiciarían la conectividad con otras áreas similares, permitiendo así el paso de animales como aves y mamíferos, así como la propagación de las plantas. Con la conectividad de estos corredores biológicos, el intercambio de especies será mayor debido a la protección que proporciona la cobertura boscosa.

\section{Sistemas de producción tradicional indígena}

Los bribris de la Alta Talamanca, en el sur de Costa Rica, han establecido una región estratégica desde la temática del uso y de la conservación de los recursos forestales y de la diversidad cultural. Cabe mencionar que los medios de vida de la población local se basan, en gran medida, en el capital natural que sostienen sus actividades agroforestales y en el capital social reflejado en diversas representaciones de organización local y relaciones con el mundo externo ${ }^{20}$.

El sistema de producción indígena procura esencialmente el sustento básico de la familia, específicamente, bajo una visión cultural y espiritual. Los sistemas de producción agrícola, dentro de los sistemas de producción tradicional indígena, combinan varias actividades bajo un plan económico de gestión comunal de los recursos naturales. Por lo tanto, «la sostenibilidad y el crecimiento pueden estar determinados por las extensiones de las áreas de cultivo, por la diversidad de la producción y por las necesidades del consumo» ${ }^{21}$.

17. Organización de las Naciones Unidas para la Alimentación y la Agricultura, Conjunto de herramientas para la gestión forestal sostenible (GFS) (2019), acceso: 16 de marzo, 2020, http://www.fao.org/ sustainable-forest-management/toolbox/modules/agroforestry/basic-knowledge/es/

18. Syed Rahman, «Agroforestería: ¿por qué los agricultores no siembran más árboles? Los bosques en las noticias», (2018), acceso: 16 de marzo, 2020, https://forestsnews.cifor.org/55793/ agroforesteria-por-que-los-agricultores-no-siembran-mas-arboles?fnl=es

19. David Kaimowitz, «Biodiversidad y agroforestería» (2012), acceso: 16 de marzo, 2020, http://www.una. ac.cr/ambi/Ambien-Tico/121/Resenas.htm

20. Candela et al., Revista Agroforestería en las Américas, 57: 85-92.

21. NITLAPLAN, Estrategias de adaptación al medio del Huracán Félix: Los casos de Butku y Awas Tingni, 2010, en Enrique Cordón, «Sistema productivo de la comunidad indígena miskitu de Tasbarrata, Raan», Revista Ciencia e interculturalidad 13, n. $^{\circ} 2$ (2013): 80-81. 


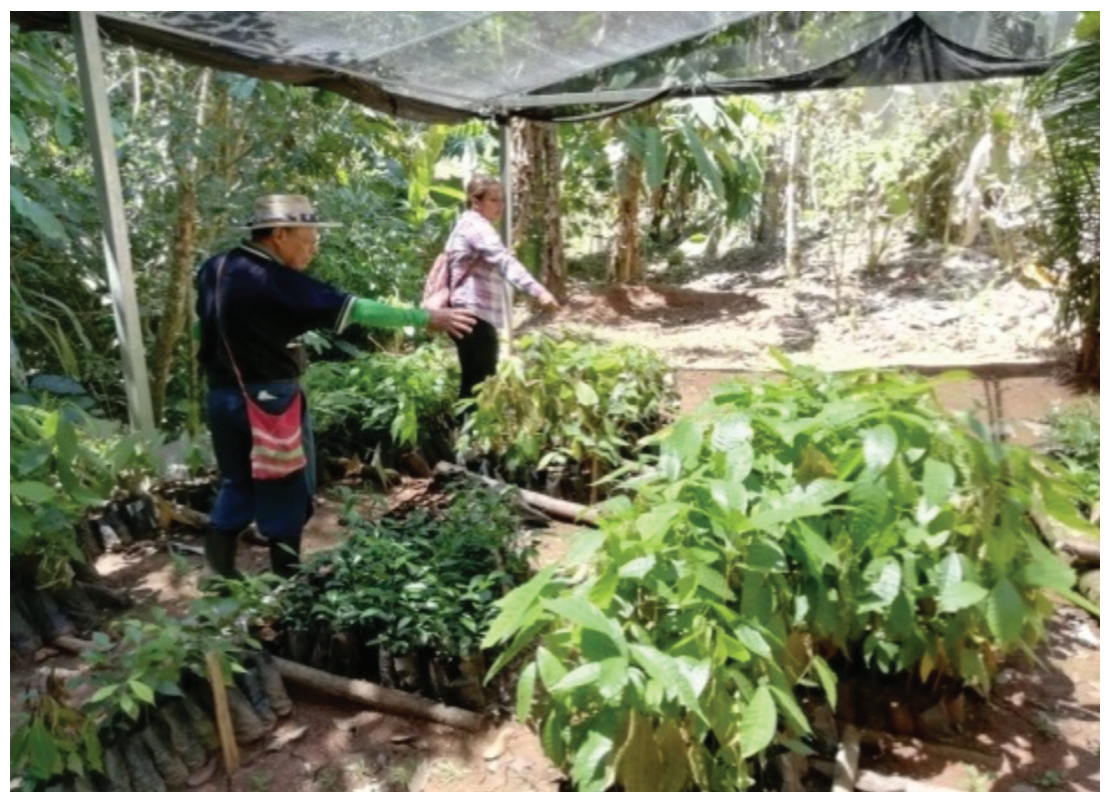

Figura 2. Vivero forestal comunitario, en el territorio indígena de Salitre. (Fotografía de la autora)

Así, en los sistemas de producción indígena se visualiza la construcción del futuro al poner en práctica las enseñanzas ancestrales e históricas de uso y conservación de los recursos de la naturaleza. Entonces, los bosques atesoran el mundo cosmológico de los habitantes nativos, por ello todo el bosque es la vida de la comunidad indígena, en el sentido espiritual, material y cultural. Esto conlleva a la sostenibilidad de los ecosistemas en el tiempo ${ }^{22}$. Por lo tanto, estos sistemas indígenas se asocian estrechamente a áreas de conservación, reservas de vida silvestre y bosques agrícolas, que buscan mantener el equilibrio ecológico y el bienestar colectivo.

\section{Agroforestería social y desarrollo sostenible en el territorio indígena}

Si de agroforestería social se habla, se puede decir que esta «comprende los sistemas de manejo de recursos, prácticas y usos de la tierra, enmarcados en una percepción cultural y filosófica, tendiente a incorporar los recursos silvestres dentro de las opciones productivas sostenibles de una unidad de manejo» ${ }^{23}$.

Ahora bien, se debe tener claro que el país cuenta con una serie de incentivos para las plantaciones forestales; sin embargo, existe un desabastecimiento de madera producto de estatutos que han repercutido en la reducción de plantaciones forestales, se ha dado una

22. Enrique Cordón, «Sistema productivo de la comunidad indígena miskitu de Tasbarrata, Raan», Revista Ciencia e interculturalidad 13, n. $^{\circ} 2$ (2013): 80-81.

23. Rodrigo Arce, Agroforestería: Un nuevo enfoque, en: Curso Agroecología, San José, 1993, en Rodrigo Arce y Alfredo García, «La agroforestería social, un nexo entre la conservación y el desarrollo sostenible», Revista Agroforestería en las Américas, 4 (s.f): 16. 
desestimulación en la industria forestal y se ha reducido el manejo de bosques naturales con fines maderables, así como las respuestas de control sobre la corta ilegal han sido limitados $^{24}$. Como resultado, el territorio indígena de Salitre y el PILA se están viendo afectados por la fuerte demanda local por madera, lo que está poniendo en peligro el capital natural y, por ende, los medios de vida de las familias indígenas.

La madera que se ha comercializado en forma clandestina figura como uno de los problemas principales para los líderes indígenas, puesto que ello incide negativamente en los intereses de las organizaciones indígenas y varios de los capitales de la comunidad: en lo referente al capital social, la legitimidad del control sobre sus recursos natrales; en cuanto al financiero y físico, se percibe el crecimiento de la ebanistería; mientras que en lo referente al capital natural y financiero, se evidencia el empobrecimiento de los recursos forestales de los usuarios directos ${ }^{25}$.

Con sustento en lo anterior, es posible agregar que la agroforestería puede ser una alternativa viable para afrontar la problemática vivida con respecto a los recursos forestales existentes. Con la implementación de esta, se incidiría en el manejo sostenible de los componentes forestales y, del mismo modo, se apoyaría el mejoramiento de los medios de vida de la comunidad rural, al proveerlas de alimento, forraje y productos de los árboles, lo que aumentaría la seguridad alimentaria y calmaría la pobreza ${ }^{26}$.

En este mismo orden de ideas, cabe mencionar que, durante décadas, la inseguridad alimentaria y la pobreza han dificultado la vida en las comunidades rurales, también se ha obstaculizado el logro del desarrollo sostenible, específicamente en los países en desarrollo. Ahora bien, la mayoría de los campesinos pobres son pequeños productores que practican una producción agrícola de bajos insumos ${ }^{27}$; es aquí donde la agroforestería puede contribuir a mejorar los medios de vida de los habitantes de escasos recursos de las zonas rurales (como Salitre), mediante la producción de alimentos (frutas, hojas comestibles, savia, miel, forraje, madera, leña, fibras textiles y medicinas). La adopción de la agroforestería puede ahorrar tiempo en la extracción de forraje y de leña, lo que supone un beneficio importante para las mujeres ${ }^{28}$.

En sus diferentes modalidades, los sistemas agroforestales constituyen buenas alternativas económicas, sociales y ambientales, puesto que contribuyen a una serie de beneficios como el aumento en la producción a nivel de finca, empleo, mejora del ingreso de los productores rurales, aumento de la fertilidad de los suelos, reducción de la erosión mediante la aportación de materia orgánica, conservación del agua al favorecer la

24. Organización de Estudios Tropicales, El abastecimiento sostenible de madera en Costa Rica (San José: CATIE, 2008).

25. Candela et al., Revista Agroforestería en las Américas, 57: 85-92.

26. Karen Arauz, «Contribución de los sistemas agroforestales en la sostenibilidad del servicio ecosistémico hídrico en las cuencas de Costa Rica», Revista AgroInnovación en el Trópico 1, n. ${ }^{\circ} 1$ (2018): 78-84.

27. María Corella, «Agroforestería y biodiversidad: La importancia de los sistemas agroforestales en la conservación de especies», Revista Biocenosis 30, n. ${ }^{\circ}$ 1-2 (2016): 59-62.

28. Organización de las Naciones Unidas para la Alimentación y la Agricultura, Agroforestería para la restauración del paisaje: Mecanismos para la restauración de bosques y paisajes (2017), acceso: 16 de marzo, 2020, http://www.fao.org/3/b-i7374s.pdf 
infiltración y reducir la escorrentía superficial que contaminan los cursos de agua, captura de carbono y conservación de la biodiversidad en ecosistemas fragmentados, como es el caso de Salitre ${ }^{29}$

\section{Especies vegetales como índice de conservación e interacción cultural, como opción productiva y como alternativa a la seguridad allimentaria}

Para los pobladores de Salitre, el llevar a cabo técnicas agroforestales les permitiría combinar especies con requerimientos ambientales diversos, lo que incidiría en mejorar el aprovechamiento de la energía radiante, ya que se estaría utilizando el espacio vertical y horizontal ${ }^{30}$. Ahora bien, si la diversidad de los sistemas agroforestales aumenta, las fincas dentro del territorio indígena asemejarían más las condiciones naturales de bosque, con lo cual el recurso suelo se aprovecharía mejor y se favorecerían los ciclos biogeoquímicos, esto debido a que se utilizan plantas con diferentes capacidades de absorción de nutrimentos y con raíces que penetren a diferentes profundidades en el perfil del suelo; aparte de ello, diversos árboles que se emplean en estos sistemas, son fijadores de nitrógeno, lo que es sumamente importante en la actualidad ${ }^{31}$.

En sentido amplio, resulta pertinente entender que los sistemas agroforestales no figuran como una alternativa que sustituya a diversos tipos tradicionales de uso de la tierra, sino más bien otra de las diferentes posibilidades que tiene a disposición el ser humano actualmente ${ }^{32}$. Por lo tanto, la implementación de un sistema específico agroforestal en Salitre, va a depender de los contextos ambientales y económicos que condicionaría el máximo aprovechamiento de la potencial productividad del sistema, sin detrimento del ambiente.

Por consiguiente, resulta de suma importancia mencionar ciertas especies vegetales que podrían ser fundamentales para establecerlas en un arreglo agroforestal en fincas de Salitre, tal es el caso del cacao (Theobroma cacao), especie que figura como uno de los elementos más importantes de la cultura indígena bribri; según la cosmovisión indígena talamanqueña, esta contiene el alma, el espíritu de la mujer que eligió el dios Sibö como su compañera, su esposa. Él la materializó en una planta con el propósito de que alimentara a sus hijos y a sus hijas, con la bebida de su fruto, el chocolate ${ }^{33}$. Entonces, al proponer una especie como el cacao en sistemas agroforestales, contribuye a mantener el sistema de producción agroecológico talamanqueño, así como a incidir en la sostenibilidad cultural del territorio indígena de Salitre.

29. Roberto Aguilera, «Agroforestería una propuesta para el desarrollo sostenible de la agricultura en la cordillera Chongon Colonche-Ecuador» (2016), acceso: 16 de marzo, 2020, http://www.eumed.net/rev/ delos/25/agroforesteria.html

30. Arauz, Revista AgroInnovación en el Trópico, 1: 78-84.

31. Luis Fournier, «Importancia de los sistemas agroforestales en Costa Rica», Revista Agronomía Costarricense 5, n. ${ }^{\circ}$ 1-2 (1981): 141-147, acceso: 16 de marzo, 2020, https://www.mag.go.cr/rev_agr/v05n12_141.pdf

32. Ibíd.

33. Rodolfo Fallas, Cultivo de Cacao Orgánico en Sistema Agroforestal para Asociación de Productores Vegas Las Palmas, Sixaola, Talamanca-Limón (31-BID) (2012), acceso: 16 de marzo, 2020, http://www. mag.go.cr/acerca_del_mag/programas/sixaola-proy31-BID-Productores_Vegas_las_Palmas.pdf 
En este mismo orden de ideas, se debe destacar que el cacao ofrecería ventajas ecológicas al ser un cultivo sostenible que incorpora muchos factores, entre estos: la captura de $\mathrm{CO}^{2}$, el cual podría permitir que la finca sea sujeta al pago de servicios ambientales, así como contribuir en la protección de los suelos de la finca, favorecería el reciclaje de nutrientes y brindaría un ambiente propicio para el desarrollo de los microorganismos del suelo ${ }^{34}$. Además, el cultivo de esta especie permitiría integrar a la familia indígena en su producción, proceso e industrialización, sin mencionar que debido al grado de variabilidad de especies en el cacaotal, se crearía un ambiente adecuado para la protección y conservación de la flora y fauna nativa.

Por otro lado, una especie como el pejibaye (Bactris gasipaes) resulta fundamental para que forme parte de un sistema agroforestal, puesto que los indígenas de Salitre utilizan el pejibaye como producto básico en su dieta diaria y con este han dado lugar a mayor diversidad de subproductos, muchos de los cuales están aún en proceso de desarrollo. Ahora bien, a dicha especie se le suma la yuca (Manihot esculenta), que también supone ser un producto alimenticio fundamental en la dieta de la familia indígena. El cultivo de la yuca tiene una gran importancia para la seguridad alimentaria y permitiría que las familias puedan generar ingresos con su venta, tanto sus raíces como sus hojas pueden ser aprovechadas para el consumo humano, lo que supondría una mayor fuente de alimento ${ }^{35}$.

Especies forestales como el cedro (Cedrela odorata) y el laurel (Cordia alliodora) son abundantes en la vegetación primaria y secundaria presente en Salitre, por lo que estas pueden servir de asocio a otras con el fin principal de obtener madera, lo que a largo plazo supondría un ingreso para las familias del territorio y de servir de sombra para otras especies como el cacao, así como incidir en la protección de esta con el fin de buscar un mejor rendimiento productivo en las cosechas.

Para finalizar, los Sistemas Agroforestales (SAF) serían una opción muy viable para ir en búsqueda del desarrollo sostenible, lo que resulta fundamental en Salitre. Si se habla del tema ambiental, los SAF proveerían hábitat y recursos para especies de plantas y animales e incidirían en el mantenimiento de la conectividad del paisaje (facilitando el movimiento de animales, semillas y polen), entre el territorio indígena (zona de amortiguamiento) y área protegida (PILA) y, de este modo, también incidirían en que el paisaje resulte menos severo para las especies forestales al reducir la frecuencia e intensidad de incendios, lo que disminuye también los efectos de bordes en los fragmentos forestales remanentes ${ }^{36}$.

\footnotetext{
34. Ibíd.
}

35. Joseneidy Nonata de Oliveira Pinheiro, «Contribución de los sistemas agroforestales a la seguridad alimentaria (con énfasis en huertos caseros) de la población del Pólo Agroforestal Wilson Pinheiro en Rio Branco, Acre» (tesis de maestría, CATIE, 2013), 116, http://repositorio.bibliotecaorton.catie.ac.cr/bitstream/handle/11554/4685/Contribucion_de_los_sistemas.pdf;jsessionid=85D8CB04F307AAC45C717D D584F3953F?sequence $=1$

36. John Beer et al., «Servicios ambientales de los sistemas agroforestales», Revista Agroforestería en las Américas, 10 (2003): 85-92. 
En cuanto al ámbito social, se debe destacar que los SAF proporcionarían un aumento en la producción, así como un mejoramiento de la salud y la nutrición de una población de escasos recursos como lo es Salitre, esto gracias a la productividad y la diversidad de productos. Asimismo, cuando la oferta de trabajo cambia en los hogares o la comunidad, por ejemplo, debido a la emigración estacional de los hombres, la agroforestería ofrece opciones que permiten aprovechar al máximo los productos por insumo de trabajo y la perpetuación de las prácticas agroforestales ayudaría a mantener los vínculos sociales establecidos mediante acuerdos de ayuda mutua ${ }^{37}$.

En lo que al ámbito económico respecta, los SAF permitirían incrementar o mantener la producción y productividad de los sistemas agrícolas, así contribuirían a reducir los insumos y los costos de producción; también incidirían en la diversificación de la producción mediante el aprovechamiento de los árboles y otras especies leñosas perennes, con el fin de producir alimentos, forraje, madera y, por ende, a la reducción de la pobreza dentro del territorio indígena, mediante el incremento de la producción en las fincas agrícolas ${ }^{38}$.

\section{Conclusiones}

Los sistemas agroforestales figuran como una opción viable para el progreso del territorio indígena de Salitre, ya que proporcionan beneficios factibles y reales a los diferentes sistemas de producción, por esto es necesario hacer un llamado a la población y promover la participación de los pobladores en este tipo de actividades como alternativa de desarrollo sostenible e incentivar su práctica.

Si se pretenden implementar sistemas agroforestales, se debe contemplar el uso del cacao como especie principal, puesto que esta figura como un producto fundamental en la cultura de los bribris de Salitre.

La incorporación de la agoroforestería en Salitre busca que, al introducir especies forestales en el sistema productivo de los bribris, se pueda incidir en el incremento de las áreas boscosas y, por ende, reducir la presión sobre los bosques naturales existentes, que son deforestados en la búsqueda de recursos forestales maderables y no maderables; mientras que la incorporación de especies comestibles pretende contribuir en la seguridad alimentaria de las familias, así como en mejorar sus medios de vida mediante la posible venta de productos (madera, frutos).

37. Organización de las Naciones Unidas para la Alimentación y la Agricultura, «Conjunto de herramientas para la Gestión Forestal Sostenible», acceso: 16 de marzo, 2020, http://www.fao.org/sustainable-forest-management/toolbox/modules/ci-a-tool-for-enhancing-sfm-from-policy-to-practice/ tools/es/

38. Ibíd. 


\title{
ABSTRACT
}

\section{Agroforestry as alternative for sustainable development in the indigenous territory of Salitre, buffer zone of La Amistad International Park}

\begin{abstract}
This article considers that agroforestry closes the gap between conservation and sustainable development in the rural community of Salitre; therefore, it could be renamed as social agroforestry. This indigenous territory lacks employment that leads to extreme poverty. Moreover, there is an inappropriate land use such as burning for weed control as well as the conflicts between the indigenous and the non-indigenous groups. It is noted the necessity to establish agroforestry systems in the area due to the fact that this production model is a better alternative for rural development in the broadest sense. The article also highlights the traditional indigenous production systems, since it argues that the Bribri from the southern part of Costa Rica founded a region based on the use and conservation of the forest resources and cultural diversity. Social agroforestry is key to understanding the natural resources management systems, practices and land use from a cultural perspective. Finally, some plant species that grow in Salitre can be used as measure of conservation and cultural integration, as alternative production and ways to attain food safety.
\end{abstract}

Key words: Agroforestry systems, protected area, cultural integration, food safety, conservation.

\section{RÉSUMÉ}

\section{Agroforesterie comme alternative du développement durable dans le territoire indigène de Salitre, zone de l'aire d'adhésion du Parc Internacional de la Amistad}

Cet article propose que l'agroforesterie ferme l'écart entre la conservation et le développement rural durable à Salitre, et par conséquent, elle peut être renommée «agroforesterie sociale». Dans ce territoire indigène il y a un manque de travail qui conduit à la pauvreté extrême. En outre, il existe des pratiques inappropriées dans l'utilisation des terres, telles que le désherbage au feu ainsi que le conflit entre les peuples indigènes et non indigènes. Ceci met en évidence la nécessité d'établir des systèmes agroforestiers dans la zone parce que ce type de modèle de production est une meilleure alternative pour le développement rural au sens large. L'article souligne aussi les systèmes traditionnels de production indigène en argumentant que les «Brisbris» du sud-est du Costa Rica ont fondé la région basée sur l'usage et la conservation des ressources naturelles et la diversité culturelle. L'agroforesterie sociale est clé pour comprendre: les systèmes de gestion de ressources naturelles, les pratiques et les usages de la terre, du point de vue culturel. Finalement, plusieurs espèces de plantes qui poussent à Salitre peuvent être employées comme indice de conservation et d'interaction culturel, ainsi que comme manière de production et un moyen d'atteindre la sécurité alimentaire.

Mots clés: Systèmes agroforestiers, zone protégée, intégration culturelle, sécurité alimentaire, conservation.

\section{Referencias bibliográficas}

Aguilera, Roberto. Agroforestería, una propuesta para el desarrollo sostenible de la agricultura en la cordillera Chongon Colonche-Ecuador. Ecuador: Universidad Tecnológica ECOTEC, 2016. Acceso: 16 de marzo, 2020. http://www.eumed.net/rev/delos/25/agroforesteria.html

Arauz-Vásquez, Karen. «Contribución de los sistemas agroforestales en la sostenibilidad del servicio ecosistémico hídrico en las cuencas de Costa Rica». Revista AgroInnovación en el Trópico 1, n. ${ }^{\circ} 1$ (2018): 78-84.

Arce Rodrigo. Agroforestería: Un nuevo enfoque. En: Curso Agroecología, San José, 1993. En Rodrigo Arce y Alfredo García. «La agroforestería social un nexo entre la conservación y el desarrollo sostenible». Revista Agroforestería en las Américas, 4 (s.f):16. 
Arce, Rodrigo y Alfredo García. s.f. «La agroforestería social un nexo entre la conservación y el desarrollo sostenible». Revista Agroforestería en las Américas, 4 (s.f): 16.

Beer, John, Celia Harvey, Muhammad Ibrahim, Jean Harmand, Eduardo Somarriba. y Francisco Jiménez. «Servicios ambientales de los sistemas agroforestales». Revista Agroforestería en las Américas, 10 (2003): $85-92$.

Borge, Carlos. El policultivo indígena de Talamanca y la conservación de la naturaleza. Heredia: INBio, 2011. Acceso: 16 de marzo, 2020. https://espanol.free-ebooks.net/ebook/ El-Policultivo-Indigena-de-Talamanca-y-la-Conservacion-de-la-Naturaleza/pdf?dl\&preview

Campregher, Christoph. «Conservación de la diversidad bio-cultural en Costa Rica: Comunidades indígenas y el ambiente». Revista Cuadernos de Antropología 21, n. ${ }^{\circ} 14$ (2011): 3-20.

Candela, Sandra, Dietmar Stoian, Eduardo Somarriba y Marilyn Villalobos. «Formas y efectos de la gobernanza forestal en los territorios indígenas bribri y cabécar de Alta Talamanca, Costa Rica». Revista Agroforestería en las Américas, 57 (2013): 85-92.

Cordón, Enrique. «Sistema productivo de la comunidad indígena miskitu de Tasbarrata, Raan». Revista Ciencia e interculturalidad 13, n. $^{\circ} 2$ (2013): 80-81.

Corella, María. 2016. «Agroforestería y biodiversidad: La importancia de los sistemas agroforestales en la conservación de especies». Revista Biocenosis 30, n. ${ }^{\circ}$ 1-2 (2016): 59-62.

Fallas, Rodolfo. 2012. Cultivo de Cacao Orgánico en Sistema Agroforestal para Asociación de Productores Vegas Las Palmas, Sixaola, Talamanca-Limón (31-BID). Acceso: 16 de marzo, 2020. http://www.mag.go.cr/ acerca_del_mag/programas/sixaola-proy31-BID-Productores_Vegas_las_Palmas.pdf

Fomento para el Medio Ambiente Mundial. s.f. «Comunidades indígenas y biodiversidad». Acceso: 16 de marzo, 2020. https://www.thegef.org/sites/default/files/publications/Indigenous-People-Spanish-PDF_0.pdf

Fournier, Luis. «Importancia de los sistemas agroforestales en Costa Rica». Revista Agronomomía Costarricense 5, n. ${ }^{\circ} 1-2$ (1981): 141-147.

Fusel, Johannes y Dinorah Sandino. s.f . Establecimiento de sistemas agroforestales en asocio con cacao. Acceso: 16 de marzo, 2020. http://www.fundesyram.info/biblioteca.php?id=3174

Guevara Víquez, Federico. «Cronología básica de los pueblos indígenas de Costa Rica: Desde los inicios del siglo XVI hasta el año 2000». San José, Costa Rica: Comisión Siwä Pakö, 2011. Acceso: 16 de marzo, 2020. https://es.scribd.com/doc/77239956/Cronologia-Basica-de-Los-Pueblos-Indigenas-CR

Kaimowitz, David. «Biodiversidad y agroforestería», 2012. Acceso: 16 de marzo, 2020. http://www.una.ac.cr/ ambi/Ambien-Tico/121/Resenas.htm

León, Krissya. Descuido en zona indígena de Salitre sigue sumando décadas. CRHoy. com, 03 de marzo, 2018. Acceso: 16 de marzo, 2020. https://www.crhoy.com/nacionales/ descuido-en-zona-indigena-salitre-sigue-sumando-decadas/

Martínez-Castillo, Roger. «La estructura cultural indígena en Talamanca». Revista Praxis, 57 (2004): $42-47$.

NITLAPLAN. Estrategias de adaptación al medio del Huracán Félix: Los casos de Butku y Awas Tingni, 2010. En Enrique Cordón, «Sistema productivo de la comunidad indígena miskitu de Tasbarrata, Raan», Revista Ciencia e interculturalidad 13, n. ${ }^{\circ} 2$ (2013): 80-81.

Nonata de Oliveira Pinheiro, Joseneidy. «Contribución de los sistemas agroforestales a la seguridad alimentaria (con énfasis en huertos caseros) de la población del Pólo Agroforestal Wilson Pinheiro en Rio Branco, Acre». Tesis de maestría. CATIE, 2013. Acceso: 16 de marzo, 2020. http://repositorio.bibliotecaorton. catie.ac.cr/bitstream/handle/11554/4685/Contribucion_de_los_sistemas.pdf;jsessionid=85D8CB04F307A AC45C717DD584F3953F?sequence $=1$ 
Organización de Estudios Tropicales. El abastecimiento sostenible de madera en Costa Rica. San José: CATIE, 2008.

Organización de las Naciones Unidas para la Alimentación y la Agricultura. Conjunto de herramientas para la gestión forestal sostenible (GFS), 2019. Acceso: 16 de marzo, 2020. http://www.fao.org/ sustainable-forest-management/toolbox/modules/agroforestry/basic-knowledge/es/

Organización de las Naciones Unidas para la Alimentación y la Agricultura. Agroforestería para la restauración del paisaje: Mecanismos para la restauración de bosques y paisajes, 2017. Acceso: 16 de marzo, 2020. http://www.fao.org/3/b-i7374s.pdf

Rahman, Syed. 2018. «Agroforestería: ¿por qué los agricultores no siembran más árboles? Los bosques en las noticias». Acceso: 16 de marzo, 2020. https://forestsnews.cifor.org/55793/ agroforesteria-por-que-los-agricultores-no-siembran-mas-arboles?fnl=es

Rivera, Ernesto. «Territorio indígena de Salitre». Semanario Universidad, 09 de julio, 2014. Acceso: 16 de marzo, 2020. https://historico.semanariouniversidad.com/page/322/?q=como-fazer-convites-gratisGRAFICA-DOS-CONVITES-como-imprimir-convites-de-casamento $\& n=22514 \& p=C u l t u r a \& j w$ source $=\mathrm{cl}$

Silva de la Fuente, Edgar, Vera L. Salazar Espinosa y Hugo Lázaro Estrada. «Información sobre los pueblos indígenas de Costa Rica como insumo para el Proyecto Regional de Manejo Integrado de Ecosistemas por Pueblos Indígenas y Comunidades de Centroamérica». Cooperación Técnica ATN-JF7695-BID, 2003. Acceso: 16 de marzo, 2020. http://pueblosindigenas.odd.ucr.ac.cr/images/documentos/ pdf/UNPAN027540.pdf

Sistema Nacional de Áreas de Conservación. Plan de Manejo Parque Internacional La Amistad-Talamanca. San José: MINAET, 2012. 